Professor Dr. Matthias Rossi, Augsburg*

\title{
Staatliche Daten als Informationsrohstoff
}

Neben besonderen Zugangsansprüchen sichert namentlich das Informationsfreiheitsrecht die Zugänglichkeit von Informationen der öffentlichen Hand. Deren Qualität erlaubt zwar grundsätzlich eine Kontrolle der Verwaltung, ist für weitergehende Zwecke aber häufig ungenügend. Insofern ist Raum für einen Markt, auf dem private Dienstleister die staatlichen Daten als Rohstoff nutzen und sie entsprechend der Nachfrage zu Informationen veredeln.

\section{Zugänglichkeit staatlicher Daten}

Knapp zwanzig Jahre nach Inkrafttreten des ersten Umweltinformationsgesetzes hat sich das Informationsfreiheitsrecht ${ }^{1}$ auch in Deutschland - von wenigen weißen Flecken abgesehen durchgesetzt. Es ist freilich ausgesprochen unübersichtlich, denn im föderal und funktional geteilten Gewaltensystem sind es verschiedene Bestimmungen, die die Zugänglichkeit amtlicher Informationen regeln. Im Ergebnis sichern drei besondere Bereiche des Informationsfreiheitsrechts die Zugänglichkeit amtlicher Informationen, neben denen das allgemeine Informationsfreiheitsrecht steht: Das Umweltinformationsrecht, das ursprünglich nur in Umsetzung einer Umweltinformationsrichtlinie der EU, mittlerweile auch in Umsetzung der völkerrechtlichen Arhus-Konvention erlassen wurde und aus kompetenzrechtlichen Gründen auf ein Umweltinformationsgesetz des Bundes sowie auf 16 Umweltinformationsgesetze der Länder verteilt ist; das Verbraucherinformationsrecht, das im Unterschied zum Umweltinformationsrecht (bislang) weder europa- noch völkerrechtlich determiniert ist und aufgrund einer umfassenden Bundeskompetenz in einem einzigen Verbraucherinformationsgesetz (sowie im Lebens- und Futtermittelgesetzbuch) geregelt ist; sowie jüngst das Geodatenzugangsrecht, das in Umsetzung der INSPIRE-Richtlinie der EU² im Geodatenzugangsgesetz des Bundes sowie in den Geodateninfrastrukturgesetzen der Länder verankert ist. Wo diese besonderen Regelungen nicht anwendbar sind, weil weder Zugang zu Umwelt- noch zu Verbraucherinformationen

* Der Autor ist Inhaber des Lehrstuhls für Staats- und Verwaltungsrecht, Europarecht sowie Gesetzgebungslehre an der Juristischen Fakultät der Universität Augsburg. Der Beitrag gibt einen Aspekt des Vortrags wieder, den der Verfasser am 1. März 2013 auf der Tagung Kultur im Wandel. Informationen der öffentlichen Hand: Zugang und Nutzung am KIT in Karlsruhe gehalten hat.

1 Zur Terminologie vgl. Rossi, in: Ehlers/Fehling/Pünder (Hrsg.), Besonderes Verwaltungsrecht, Bd. $2,3$. Aufl. 2013, § 63 Rn. 3.

2 Richtlinie 2007/2/EG vom 14. 3. 2007 zur Schaffung einer Geodateninfrastruktur in der Europäischen Gemeinschaft (INfrastructure for SPatial Information in the European Community), ABl. vom 25. 4. 2007 Nr. L 108. 
oder zu Geodaten begehrt wird, greift das allgemeine Informationsfreiheitsrecht. ${ }^{3}$ Entsprechend der föderalen Kompetenzverteilung ist es in einem Informationsfreiheitsgesetz des Bundes sowie mittlerweile in elf Landesgesetzen geregelt. ${ }^{4}$

Die Unterschiede des Informationsfreiheitsrechts zu rechtsstaatlich begründeten Ansprüchen auf Auskunft und Akteneinsicht sind mittlerweile hinlänglich beschrieben worden. Betont werden soll hier nur, dass der Informationszugang nach dem Informationsfreiheitsrecht relativ kostengünstig ist. Zwar werden die Kosten für Gebühren und Auslagen aus politischer Perspektive z. T. immer noch als recht hoch oder gar als prohibitiv empfunden. Doch einfache Auskünfte sind stets kostenfrei ${ }^{5}$ und der Wert der Information spielt entgegen dem allgemeinen gebührenrechtlichen Äquivalenzgrundsatz keine Rolle, denn die Gebührenordnungen deckeln die Gebührenhöhe bei 500,- Euro. ${ }^{6}$

\section{Nutzung der zugänglichen Daten}

Von der Frage, ob und mit welchen Ausnahmen Daten der öffentlichen Hand zugänglich sind, ist die Frage zu trennen, wie diese Daten genutzt werden dürfen.

\section{Indifferenz des Informationsfreiheitsrechts}

Das Informationsfreiheitsrecht selbst ist insoweit indifferent, es verhält sich nicht zur Verwendung der zugänglichen Daten. Weder statuiert es positiv eine bestimmte Verwendung noch etabliert es Schranken für bestimmte Nutzungsarten.

\section{Intentionen des Informationsfreiheitsrechts}

Allerdings verfolgt das Informationsfreiheitsrecht ausweislich der jeweiligen Gesetzesbegründungen verschiedene Zielsetzungen, die nicht bei der Gewährleistung der Zugänglichkeit, also bei der Herstellung von Transparenz, enden, sondern ganz bestimmte Verwendungsmöglichkeiten vor Augen haben. Nach den Vorstellungen der Gesetzgeber sollen die Daten erstens zur Kontrolle der Verwaltung, zweitens zur Partizipation an Verwaltungsentscheidungen und drittens zu deren Akzeptanz im Sinne eines ,plébiscite de tous les jours“genutzt werden. Im

3 Eine graphische Übersicht über das Informationsfreiheitsrecht findet sich bei Rossi, in: Ehlers/Fehling/Pünder (Hrsg.), Besonderes Verwaltungsrecht, Bd. 2, 3. Aufl. 2013, § 63 Rn. 23.

4 In Baden-Württemberg und Niedersachen darf ein Informationsfreiheitsrecht nach den letzten Regierungswechseln erwartet werden.

5 Vgl. bspw. $\S 10$ Abs. 1 S. 2 IFG, $\S 12$ Abs. 1 S. 2 UIG.

6 S. etwa $\S 1$ Abs. 2 UIGKostV, Nr. 1.3 und 2.2 IFGGebV. 
besonderen Informationsfreiheitsrecht kommt eine Verwendung der Daten zugunsten des Umweltschutzes bzw. zugunsten des Verbraucherschutzes hinzu.

Insbesondere die allgemeinen Zielsetzungen der Kontrolle, der Partizipation und der Akzeptanz stehen in der Konsequenz der demokratie-theoretischen Begründung des Informationsfreiheitsrechts. Danach soll der freie Informationszugang vor allem ein Instrument für politisch motivierte und interessierte Bürger sein, um Verwaltungsentscheidungen kontrollieren bzw. im Vorfeld beeinflussen zu können. Doch noch einmal sei betont, dass sich diese Zielsetzungen nicht im Gesetzestext wiederfinden. Insofern ist es bedenklich, wenn sie im Wege der teleologischen Auslegung ein Gewicht erhalten, das in der Abwägung mit entgegenstehenden grundrechtlich geschützten Interessen zu überwiegen scheint.

\section{Grundrechtsgebrauch ermöglichende Wirkung}

Denn die Wirkungen des Informationsfreiheitsrechts gehen über diese politischen Zielsetzungen des Gesetzgebers hinaus. Das Informationsfreiheitsrecht entfaltet schlicht Grundrechtsgebrauch ermöglichende Wirkungen: Es differenziert nicht danach, ob die erlangten Informationen zu politischen oder $\mathrm{zu}$ anderen Zwecken verwendet werden, etwa $\mathrm{zu}$ wirtschaftlichen Zwecken oder auch zu Zwecken, die sich grundrechtlich allein unter die allgemeine Handlungsfreiheit fassen lassen. Das Informationsfreiheitsrecht ist insoweit indifferent. Bei der Abwägung mit privaten Belangen Dritter stehen sich insoweit zwei grundsätzlich gleich gewichtige Interessen gegenüber, ohne dass die Zugänglichkeit durch seine vermeintlich demokratische Begründung ein besonderes Gewicht erhielte. ${ }^{7}$

\section{Mittelbare Beschränkung der Nutzung}

Erkennbar wird dies daran, dass sich jedenfalls das IFG des Bundes mittelbar doch zur Nutzung der zugänglichen Informationen verhält. Denn entgegen dem Grundsatz der Voraussetzungslosigkeit stellt $\S 7$ Abs. 1 S. 3 IFG hinsichtlich solcher Informationen, die schutzwürdige Belange Dritter berühren, auf den Zweck des Informationszugangs ab. Dieser Zweck beeinflusst in der Abwägung mit entgegenstehenden Belangen Dritter maßgeblich die Frage, ob die begehrten Informationen überhaupt zugänglich sind oder nicht. Die Steuerungswirkung dieser mittelbaren Beschränkung der Zugänglichkeit und mit ihr der Nutzung ist freilich nicht sehr scharf. Denn faktisch steht es den Antragstellern frei, die einmal zugänglichen Informationen auch zu anderen als den angegebenen Zwecken zu nutzen.

7 Rossi, in: Hecker u.a. (Hrsg.), Aktuelle Rechtsfragen und Probleme des Informationszugangs, UTR 108, S. 197, $221 \mathrm{f}$. 


\section{Unzulässigkeit individueller Nutzungsbeschränkungen}

Auch rechtlich sind die Antragsteller nicht verpflichtet, die Informationen nur zu dem angegebenen Zweck zu verwenden. Denn da sich das abstrakt-generelle Informationsfreiheitsrecht nicht zur Nutzung verhält, ist es den zugangsgewährenden Behörden untersagt, die Verwendung der preisgegebenen Informationen im konkret-individuellen Fall zu beschränken. Die zugangsgewährenden Verwaltungsakte müssen insofern zwar zwischen zugänglichen und nicht zugänglichen Informationen unterscheiden, um die Zugänglichkeit so weit wie möglich zu gewähren, dürfen hinsichtlich der zugänglichen Daten aber nicht mit Auflagen oder Bedingungen hinsichtlich der Verwendung der Informationen verbunden werden. Dies widerspräche dem Grundrechtsgebrauch ermöglichenden Sinn des Informationsfreiheitsrechts. ${ }^{8}$ Gleiches gilt für vertragliche Zusatzvereinbarungen zur beschränkten Nutzung der Daten. Sie sind unzulässig, weil sie der Intention des Informationsfreiheitsrechts widersprechen.

Die Unzulässigkeit individueller Nutzungsbeschränkungen mag auf den ersten Blick widersinnig erscheinen. Denn es ließe sich argumentieren, dass eine Zugänglichkeit unter beschränkter Verwendungserlaubnis sowohl dem individuellen Interesse des Antragstellers als auch der gesetzlich intendierten Öffentlichkeit der Verwaltung näher komme als die Verweigerung des Zugangs aus Sorge um die Nichteinhaltung der angegeben Nutzungsverwendung. Doch eine solche Argumentation vermag nicht zu überzeugen. Sie übersieht, dass die Verwaltung mit der Preisgabe zugleich die Herrschaft über die Informationen verliert. Sie kann weder durch Nebenbestimmungen noch durch Vertrag ausschließen, dass die preisgegebenen Informationen zu anderen als den festgelegten Zwecken oder von anderen Personen als dem Antragsteller verwendet werden. Insbesondere ist nicht ausgeschlossen, dass sich die preisgegebenen Daten im Zusammenhang mit anderen Informationen zu neuem Wissen generieren. Das verwaltungsrechtliche Instrumentarium mit den Möglichkeiten der Rücknahme bzw. des Widerrufs bliebe wegen der Irreversibilität von Informationen ohne Wirkung. ${ }^{9}$ Die zweckfremde Verwendung oder unerlaubte Weitergabe von Informationen wäre allenfalls Anknüpfungspunkt für sekundäre Ansprüche, etwa auf Gegendarstellung oder Schadensersatz, holte die einmal preisgegebene Information aber nicht zurück. „Audacter calumniare, semper aliquid haeret“ - diese Wirkung von Behauptungen darf sich vielleicht die Politik oder der zweitklassige Journalismus zu eigen machen, nicht aber der Staat. Rechtliche Verwendungs- 
beschränkungen sind wegen ihrer faktischen Unwirksamkeit deshalb kein Instrument des Informationsfreiheitsrechts.

\section{Fundgrube statt Fachhandel}

$\mathrm{Zu}$ welchen Zwecken die zugänglichen Informationen genutzt werden, ist nicht zuletzt eine Frage ihrer Qualität. Das Qualitätsniveau der nach dem Informationsfreiheitsrecht zugänglichen Daten ist entsprechend seiner ideologisch überhöhten Intention auf die Kontrolle der Verwaltung, die Partizipation der Bürger sowie die Akzeptanz administrativer Entscheidungen ausgerichtet. Diesen Zwecken genügt es zumeist. Für andere, für weitergehende Verwendungszwecke erweist sich das Informationsfreiheitsrecht aus folgenden Gründen dagegen eher als Fundgrube denn als Fachhandel.

\section{Daten als Nebenprodukt der Aufgabenerfüllung}

Zunächst darf nicht übersehen werden, dass die zugänglichen Daten in der Regel nur als Nebenprodukt der Aufgabenerfüllung anfallen. Die Behörden sammeln Daten grundsätzlich nicht als Selbstzweck, sondern verwenden sie zu einem bestimmten sachlichen Zweck - im Rahmen einer Eröffnungs- oder einer laufenden Kontrolle oder im Zusammenhang mit der planenden Verwaltungstätigkeit.

Nur ausnahmsweise werden Daten gezielt zur Preisgabe erhoben, gesammelt und aufbereitet, etwa bei der Erstellung von Statistiken, ${ }^{10}$ der Erhebung von Wetterdaten ${ }^{11}$ oder der Kartographierung der Meere durch das BSH. ${ }^{12}$ Auch die Fachinformationszentren sind ein Beispiel für die gezielte Ansammlung und Aufbereitung durch den Staat, wenngleich hinsichtlich der Rechtsform, der Trägerschaft und der Geschäftsmodelle zwischen den Zentren erhebliche Unterschiede bestehen. ${ }^{13} \mathrm{Ob}$, unter welchen Voraussetzungen und mit welchen Beschränkungen der Staat solche Daten bzw. aufbereiteten Informationen nach Marktprinzipien bereitstellen kann, ist keine Frage des Informationsfreiheitsrechts, sondern bestimmt sich nach allgemeinen rechtlichen Grundsätzen ${ }^{14}$ sowie vermehrt nach Maßgabe europarechtlicher Vorgaben. ${ }^{15}$ Generell ist dem Staat ein marktwirtschaftliches Agieren nicht verboten. Stets sind aber 
vor allem die Gleichheitsrechte zu beachten sowie auszuloten, inwieweit den Staat eine allgemeine und deshalb allein steuerfinanzierte Aufgabe trifft, eine angemessene informationelle Grundversorgung zu leisten. ${ }^{16}$

\section{Beschränkung auf vorhandene Informationen}

Weil die Daten bei den Behörden grundsätzlich nur als Nebenprodukt ihrer Aufgabenerfüllung anfallen, richtet sich der informationsfreiheitsrechtliche Zugangsanspruch regelmäßig nur auf vorhandene Informationen. Die Behörden trifft grundsätzlich weder eine Informationsbeschaffungs- noch eine besondere Informationsaufbereitungspflicht. Den Wünschen der Antragsteller können sie insoweit häufig nicht gerecht werden. Sie generieren und archivieren Daten nur nach ihren eigenen spezifischen Bedürfnissen und nach den Regeln der Aktenführung, nicht hingegen nach etwaigen und im Vorhinein ja auch in ihrer Vielfalt nicht vorhersehbaren Wünschen potentieller Antragsteller.

Allerdings gelten diese Grundsätze nicht durchgängig. Man wird aus rechtsstaatlichen Gründen annehmen müssen, dass die Behörde verpflichtet ist, die Daten jedenfalls in einer verständlichen, das heißt vor allen Dingen auch in einer lesbaren Form bereitzustellen. Die Behörde schuldet mindestens entsprechende technische Geräte, ggf. in sog. „reading-rooms“, um Daten sichtbar und lesbar zu machen. Eine individuelle Erläuterung der Daten schulden die informationspflichten Stellen hingegen grundsätzlich nicht. Sie sind allerdings verpflichtet, ihr Informationssystem von vornherein so anzulegen, dass sich der Bürger aus den abgelegten Daten relativ einfach eine Meinung bilden kann. Die grundsätzliche Zugänglichkeit aller Informationen ist insoweit von vornherein bei der Aktenführung zu berücksichtigen. Betreibt eine Behörde kein ordentliches Informationsmanagement, kann sie sich bei einem individuellen Zugangsantrag jedenfalls nicht auf den Ausnahmetatbestand des unverhältnismäßigen Verwaltungsaufwandes berufen.

\section{Vollständigkeit und Aktualität}

Auch bei einem ordentlichen Informationsmanagement besteht indes keine Garantie dafür, dass es sich bei den zugänglichen Daten um vollständige und aktuelle Informationen handelt. Vollständigkeit wird nur in Bezug auf den Informationsbestand der jeweiligen Behörde geschuldet. $\mathrm{Ob}$ und wo zu dem betreffenden Sachverhalt weitere Informationen existieren, wird durch die Bescheidung eines Zugangsantrags nicht abschließend beantwortet. Bewusst sein sollte den Antragstellern auch, dass sich der Inhalt und die Bedeutung von Informationen im 
Laufe der Zeit verändern können. Dies mag insbesondere bei solchen Informationen bedacht werden, die die Behörden im Rahmen einer verwaltungsrechtlichen Eröffnungskontrolle einholen und zur Grundlage ihrer Entscheidung machen.

\section{Richtigkeit der Informationen}

Das Qualitätsniveau der zugänglichen Daten leidet schließlich darunter, dass es im Informationsfreiheitsrecht keine Gewähr für die Richtigkeit der Informationen gibt. ${ }^{17}$ Anders als etwa die Richtigkeit von Grundbüchern vermutet wird und insofern ein öffentlicher Glaube besteht, ${ }^{18}$ bezieht sich die „Beweiskraft“ im Informationsfreiheitsrecht nur auf die bloße Existenz von Informationen, nicht auf deren Inhalt. Die Behörden trifft deshalb keine Amtspflicht, den Inhalt der preiszugebenden Informationen zu überprüfen, so dass ein durch eine falsche Information verursachter Schaden grundsätzlich nicht über einen Amtshaftungsanspruch ersetzt werden kann.

\section{Veredelung der Daten zu Informationen}

Trotz der niedrigen Qualität der zugänglichen Daten eignet sich das Informationsfreiheitsrecht sehr gut für die Bereitstellung des Rohmaterials, aus dem der Markt Informationen gewinnen und bereitstellen kann. ${ }^{19}$ Denn die Informationen der öffentlichen Hand, die quasi nur als Nebenprodukt der eigentlichen Aufgabenerfüllung anfallen, genügen in ihrer Qualität nur solange den Anforderungen der Antragsteller, wie primär die Kontrolle der Aufgabenerfüllung, möglicherweise auch die Akzeptanz und die Partizipation an dieser Aufgabenerfüllung, im Vordergrund des Interesses stehen. Soweit es aber darum geht, diese Informationen anderweitig zu nutzen, werden sie dem Antragsteller in der Regel nicht genügen.

\section{Sekundärer Informationsmarkt}

Hier nun eröffnet die grundsätzliche Verwendungsfreiheit der zugänglichen Informationen die Möglichkeit, die „Rohdaten“ der öffentlichen Hand zu Informationen zu veredeln, die Fundgrube in einen Fachhandel für Informationen zu verwandeln. Das Qualitätsniveau dieser Informationen bestimmt allein der Markt. Er wird zunächst herauskristallisieren, an welchen Informationen es Bedürfnisse gibt und an welchen nicht. Er wird sodann dazu beitragen, die

17 Explizit etwa $\S 7$ Abs. 3 S. 2 IFG.

18 Vgl. $\S 891$ ff. BGB.

19 Zur Qualifizierung staatlicher Informationen als Rohstoff vgl. schon Spinner, Die Wissensordnung, S. 19; Büllesbach, NJW 1991, S. 2593, 2594. 
Qualität der Information zu verbessern. Denn die privaten Anbieter können das Rohmaterial aufbereiten, sie können es zusammenstellen, anreichern und auf diese Art und Weise zu einer Informationsgewinnung beitragen.

Die - jedenfalls von der EU intendierte ${ }^{20}$ - wirtschaftliche Nutzung der Information, die Verbindung mit anderen Informationen und die daraus entstehende Generierung neuer Informationen ist eine Aufgabe, die der Markt typischerweise deutlich besser bewerkstelligen kann als die öffentliche Hand. Die öffentliche Hand sammelt, verarbeitet und archiviert Informationen zu ihren spezifischen Bedürfnissen. Doch wozu diese Informationen darüber hinaus genutzt werden können, ist eine Frage, die im besten Sinne durch Nachfrage ermittelt werden kann, auf die dann wiederum das Angebot reagieren kann. Dass private Informationsvermittler die aufbereiteten Informationen auf einem Informationssekundärmarkt regelmäßig nur gegen Entgelt zur Verfügung stellen, ist nicht nur legitim, sondern auch mit dem Grundgedanken des Informationsfreiheitsrechts vereinbar. Denn potentielle Nachfrager haben stets noch die Wahl, entweder selbst - vergleichsweise kostengünstig - auf die originären Daten der öffentlichen Hand zuzugreifen oder sie in aufbereiteter Art von privaten Informationsvermittlern zu erhalten. Dass der Preis für diese Veredelung nur schwer zu bestimmen ist, weil sich der Wert der heute gehandelten Informationen häufig erst in der Zukunft erweisen wird, steht der marktmäßigen Ausgestaltung der sekundären Informationsverteilung nicht entgegen. Vielmehr werden sich Fälle, in denen Informationen gemessen an ihrem späteren Wert günstig erworben wurden, die Waage halten mit Fällen, in denen sie zu teuer erkauft wurden.

\section{Chancen und Risiken}

Eine solche sekundäre, marktwirtschaftlich organisierte Informationsverteilung bietet Chancen, birgt aber auch Risiken.

Aus Marktperspektive ist zunächst positiv hervorzuheben, dass die Kompetenzgrenzen des Informationsfreiheitsrechts auf einem sekundären Markt überwunden werden. Die mühselige Arbeit, die Informationen der einzelnen Behörden nach den jeweiligen Gesetzen der Länder und des Bundes flächendeckend zusammenzutragen, übernehmen die privaten Dienstleister. Die skizzierte Diversifizierung und Zerfaserung des Informationsfreiheitsrechts wird jedenfalls für den Endkunden aufgehoben.

20 Vgl. schon Europäische Kommission, Grünbuch über die Informationen des öffentlichen Sektors in der Informationsgesellschaft, KOM (1998) 585. 
Damit zusammenhängend kann auf dem sekundären Informationsmarkt eine Kohärenz und Konformität der Daten erreicht werden, die auf dem staatlichen Primärmarkt wegen unterschiedlichster gesetzlicher Vorgaben und behördlicher Praktiken nicht gewährleistet ist. Verschiedene Formate, unterschiedliche Aktenführungen werden für den Endkunden aufbereitet und vereinheitlicht. Die Veredelung besteht schließlich nicht zuletzt auch darin, die Informationen überhaupt erst einmal sichtbar zu machen. Indem Informationen erschlossen und indiziert werden, werden sie suchbar und dadurch auch auffindbar.

Aus rechtsstaatlicher Perspektive ist namentlich die Kompetenzüberlagerung des sekundären Marktes mit dem Nachteil verbunden, dass sich unter Umständen auch die öffentliche Hand dieser nun verbundenen Informationen bedienen kann. Insofern droht - im Übrigen auch bei einer Anwendung durch Private - die Gefahr, dass der datenschutzrechtliche Zweckbindungsgrundsatz als besonderer Ausdruck der freiheitssichernden und -gewährleistenden Zuständigkeitsverteilung unterlaufen wird.

Gewichtiger als dieses Argument ist vor allen Dingen, dass durch den kompetenzübergreifenden Zugriff eine Rekombination solcher Informationen möglich wird, deren Zugänglichkeit nach den Ausnahmetatbeständen an sich ausgeschlossen sein soll. Denn wenn die Ausnahmetatbestände eng ausgelegt und so im Zweifel etwa Bruchstücke von Betriebs- und Geschäftsgeheimnissen oder auch personenbezogene Daten preisgegeben werden, weil bei der konkreten Entscheidung - zu Recht - angenommen wird, es sei eine hinreichende Anonymisierung erfolgt oder das Betriebs- und Geschäftsgeheimnis sei im konkreten Einzelfall nicht hoch zu bewerten, so besteht nun auf dem sekundären Markt die Möglichkeit, durch die Anreicherung mit anderen Informationen, mit zusätzlichem Wissen also, genau diejenigen Informationen zu rekonstruieren, deren Geheimhaltung grundrechtlich geboten ist. Insoweit spricht diese Möglichkeit der Rekombination, der Rekonstruktion, erneut dafür, die Ausnahmetatbestände jedenfalls dann weit zu verstehen, wenn sie dem Schutz privater Belange zu dienen bestimmt sind.

\section{Informationsaufbereitung durch den Staat}

Die Beschränkung der öffentlichen Hand auf ihre eigentliche Aufgabenerfüllung, bei deren Tätigkeit die Informationen als Nebenprodukt anfallen, und die anschließende Aufbereitung dieses Rohstoffs durch den Markt scheint viele Vorteile zu haben. Gleichwohl stellt sich erneut die Frage, ob und in welchem Umfang es dem Staat möglich ist bzw. sein sollte, diese Veredelung der Information selbst vorzunehmen. 
Soweit gegen das Agieren des Staates auf dem sekundären Informationsmarkt vorgebracht wird, der Bürger habe bereits als Steuerzahler für die Informationen des Staates bezahlt, trägt dies aus zwei Gründen nicht: Zum einen ist allenfalls der Informationsrohstoff steuerfinanziert, nicht hingegen die Veredelung, der Mehrwert, der bei der Informationsaufbereitung erzeugt wird. Zum anderen ist es durchaus geboten, jedenfalls aber legitim, zwischen den allgemeinen Kosten der Steuerzahler und dem besonderen Nutzen zu differenzieren, den ein einzelner von einer bestimmten Information hat. Dementsprechend erscheint es zulässig, dass sich der Staat nicht auf die Bereitstellung von Rohdaten beschränkt, sondern auch auf dem Informationssekundärmarkt zu einer Veredelung der Daten beiträgt. Die politischen Ziele der Kontrolle, der Partizipation und der Akzeptanz werden hiervon nicht berührt.

\section{Fazit}

Das Informationsfreiheitsrecht in Deutschland ist nach wie vor auf bestimmte Zwecke ausgerichtet, es wird namentlich als ein Instrument zur Kontrolle der Verwaltung begriffen. Damit bleibt es hinter den Möglichkeiten zurück, die die bei der öffentlichen Hand vorhandenen Informationen bieten. Immerhin steht es anderen, steht es insbesondere wirtschaftlichen Verwendungen nicht entgegen.

Unter Betonung des „Wertes“, den diese Informationen in einer Informationsgesellschaft haben oder haben können, erscheint das hier skizzierte zweistufige Modell der staatlichen Bereitstellung von Rohdaten und der privaten Veredelungen zu Informationen gleichwohl nicht alternativlos. Vielmehr lässt sich durchaus die Frage stellen, weshalb der Staat in einem aufwändigen und komplizierten Regelungssystem Informationsansprüche des Bürgers statuiert und dabei zahlreiche Folgeprobleme provoziert, anstatt den Behörden Anreize zu setzen, von sich aus ein ordentliches Informationsmanagementsystem zu betreiben. Die Aussicht, mit besseren Informationen Einnahmen zu erzielen, wäre sicherlich eine Motivation, die das Qualitätsniveau der zugänglichen Informationen erheblich verbessern könnte. Solche Informationen würden keine Kosten verursachen, sondern Preise erzielen. 